\title{
Molecular characterization of NRXN1 deletions from 19,263 clinical microarray cases identifies exons important for neurodevelopmental disease expression
}

\author{
Chelsea Lowther, BSc${ }^{1}$, Marsha Speevak, PhD², Christine M. Armour, MD, MSc ${ }^{3}$, Elaine S. Goh, MD², \\ Gail E. Graham, MD, Chumei Li, MD, PhD ${ }^{4,5}$, Susan Zeesman, MSc ${ }^{5}$, Malgorzata J.M. Nowaczyk, MD ${ }^{5,6}$, \\ Lee-Anne Schultz, MSc ${ }^{5}$, Antonella Morra, MD², Rob Nicolson, MD7, Peter Bikangaga, MD $^{8}$, \\ Dawa Samdup, MD ${ }^{9}$, Mostafa Zaazou, MD², Kerry Boyd, MD ${ }^{10}$, Jack H. Jung, MD ${ }^{11}$, Victoria Siu, MD ${ }^{12}$, \\ Manjulata Rajguru, MD ${ }^{13}$, Sharan Goobie, MD ${ }^{12}$, Mark A. Tarnopolsky, MD ${ }^{14}$, Chitra Prasad, MD ${ }^{12}$, \\ Paul T. Dick, MD ${ }^{15}$, Asmaa S. Hussain, MD ${ }^{11}$, Margreet Walinga, MD ${ }^{16}$, Renske G. Reijenga, MD ${ }^{17}$, \\ Matthew Gazzellone, MSc ${ }^{18}$, Anath C. Lionel, PhD ${ }^{18}$, Christian R. Marshall, PhD ${ }^{18}$, \\ Stephen W. Scherer, PhD ${ }^{18,19}$, Dimitri J. Stavropoulos, PhD ${ }^{20}$, Elizabeth McCready, $\mathrm{PhD}^{6}$ and \\ Anne S. Bassett, MD ${ }^{1,21}$
}

\begin{abstract}
Purpose: The purpose of the current study was to assess the penetrance of NRXN1 deletions.

Methods: We compared the prevalence and genomic extent of NRXN1 deletions identified among 19,263 clinically referred cases to that of 15,264 controls. The burden of additional clinically relevant copy-number variations (CNVs) was used as a proxy to estimate the relative penetrance of NRXN1 deletions.
\end{abstract}

Results: We identified $41(0.21 \%)$ previously unreported exonic NRXN1 deletions ascertained for developmental delay/intellectual disability that were significantly greater than in controls (odds ratio $(\mathrm{OR})=8.14 ; 95 \%$ confidence interval $(\mathrm{CI}): 2.91-22.72 ; P<0.0001$ ). Ten $(22.7 \%)$ of these had a second clinically relevant CNV. Subjects with a deletion near the $3^{\prime}$ end of NRXN1 were significantly more likely to have a second rare CNV than subjects with a $5^{\prime}$ NRXN1 deletion $(\mathrm{OR}=7.47 ; 95 \% \mathrm{CI}: 2.36-23.61 ; P=0.0006)$. The prevalence of intronic NRXN1 deletions was not statistically different between cases and controls $(P=0.618)$. The majority $(63.2 \%)$ of intronic NRXN1 deletion cases had a second rare CNV at a prevalence twice as high as that for exonic NRXN1 deletion cases $(P=0.0035)$.

Conclusions: The results support the importance of exons near the $5^{\prime}$ end of NRXN1 in the expression of neurodevelopmental disorders. Intronic NRXN1 deletions do not appear to substantially increase the risk for clinical phenotypes.

Genet Med advance online publication 19 May 2016

Key Words: copy-number variation; genotype-phenotype; NRXN1; penetrance; variable expression

\section{INTRODUCTION}

Neurexins are a group of highly polymorphic presynaptic cell adhesion molecules that primarily bind to neuroligins. ${ }^{1}$ The three neurexin genes (NRXN1, NRXN2, and NRXN3) are highly conserved and undergo extensive alternative splicing ${ }^{2}$ to produce thousands of isoforms that appear to be both spatially and temporally regulated. ${ }^{3,4}$ The two main isoforms for each neurexin gene, the longer $N R X N-\alpha$ and the shorter $N R X N-\beta$, are transcribed from two independent promoter regions and give rise to proteins with similar C-terminal regions but different N-terminal ectodomains. ${ }^{5}$ The $N R X N-\alpha$ and $N R X N-\beta$ isoforms both bind to postsynaptic neuroligins and leucinerich repeat transmembrane proteins but with varying degrees of affinity. ${ }^{1,6,7} N R X N-\alpha$ also binds to dystroglycan and cerebellin. ${ }^{89}$ NRXN1 (OMIM 600565) is the largest neurexin gene (comprising approximately $1.1 \mathrm{Mb}$ of genetic sequence) ${ }^{2}$ and the one most implicated as a top candidate gene for neurodevelopmental and neuropsychiatric conditions (Figure 1). ${ }^{10}$

\footnotetext{
${ }^{1}$ Institute of Medical Science, University of Toronto, Toronto, Ontario, Canada; ${ }^{2}$ Trillium Health Partners Credit Valley Site, Toronto, Ontario, Canada; ${ }^{3}$ Regional Genetics Program, Children's Hospital of Eastern Ontario, Toronto, ON, Canada; ${ }^{4}$ Department of Pediatrics, University of Ottawa, Ottawa, Ontario, Canada; ${ }^{5}$ McMaster Children's Hospital, Department of Pediatrics and Clinical Genetics Program, Hamilton, Ontario, Canada; ${ }^{6}$ Department of Pathology and Molecular Medicine, McMaster University, Hamilton, Ontario, Canada; ${ }^{7}$ Department of Psychiatry, Western University, London, Ontario, Canada; ${ }^{8}$ William Osler Health Centre, Brampton, Ontario, Canada; ${ }^{9}$ Hotel Dieu Hospital, Child Development Centre, Kingston, Ontario, Canada; ${ }^{10}$ Department of Psychiatry, McMaster University, Hamilton, Ontario, Canada; ${ }^{11}$ London Health Sciences Centre, Children's Hospital of Western Ontario, London, Ontario, Canada; ${ }^{12}$ Department of Pediatrics, Schulich School of Medicine and Dentistry, London, Ontario, Canada; ${ }^{13}$ Cambridge Memorial Hospital, Cambridge, Ontario, Canada; ${ }^{14}$ Department of Pediatrics, McMaster University, Hamilton, Ontario, Canada; ${ }^{15}$ Grey Bruce Health Services, Owen Sound, Ontario, Canada; ${ }^{16}$ Vanboeijen, Assen, The Netherlands; ${ }^{17}$ Ipse de Bruggen, Zwammerdam, The Netherlands; ${ }^{18}$ The Centre for Applied Genomics, the Hospital for Sick Children, Toronto, Ontario, Canada; ${ }^{19}$ McLaughlin Centre and Department of Molecular Genetics, University of Toronto, Toronto, Ontario, Canada; ${ }^{20}$ Cytogenetics Laboratory, Department of Pediatric Laboratory Medicine, the Hospital for Sick Children, Toronto, Ontario, Canada; ${ }^{21}$ Department of Psychiatry, Faculty of Medicine, University of Toronto, Toronto, Ontario, Canada. Correspondence: Anne S. Bassett (anne.bassett@utoronto.ca) Submitted 5 January 2016; accepted 16 March 2016; advance online publication 19 May 2016. doi:10.1038/gim.2016.54
} 


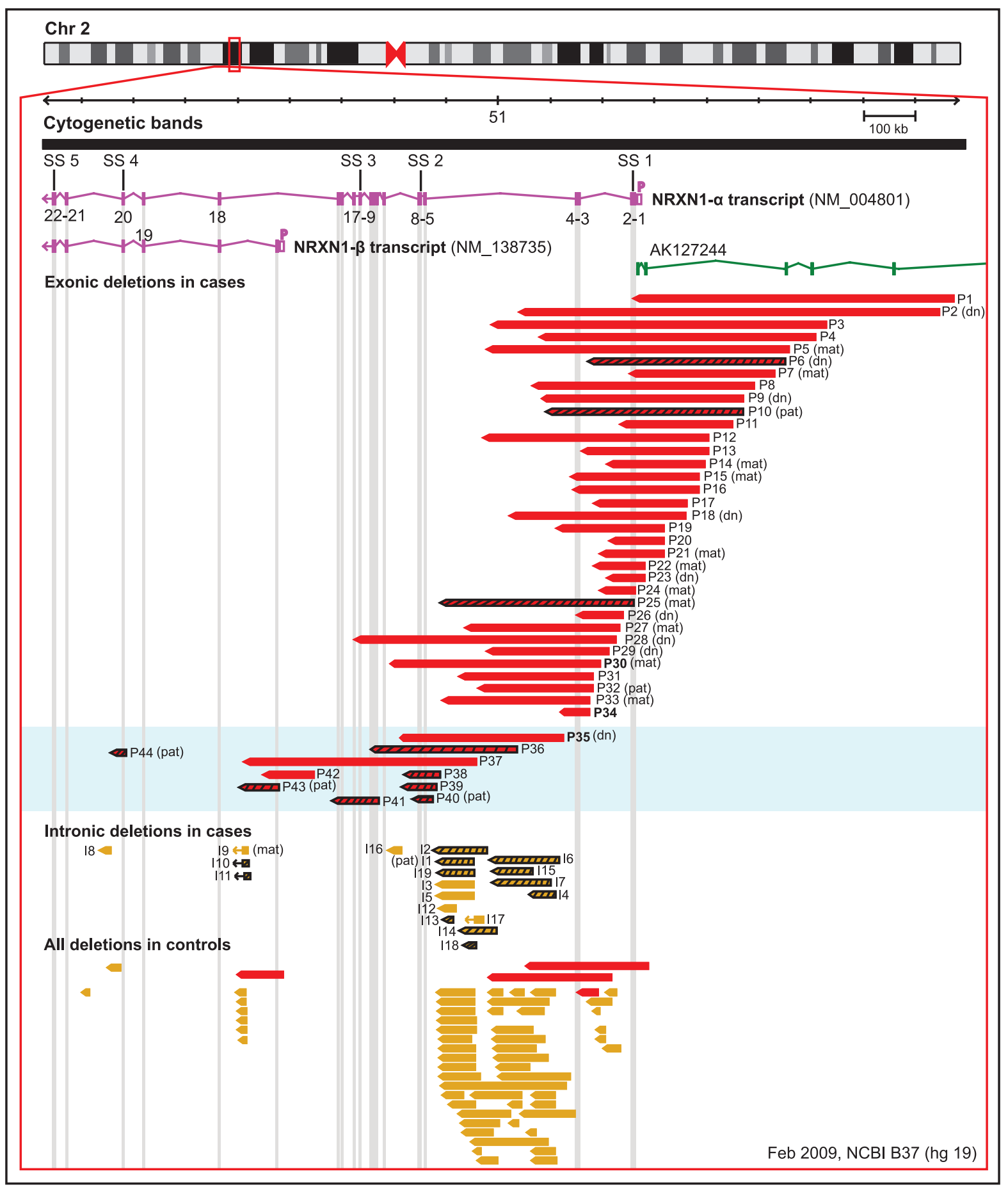

Figure 1 Novel exonic and intronic NRXN1 deletions identified in cases and controls. The image was modified from the Database of Genomic variants (http://dgv.tcag.ca), NCBI Build 37 (hg 19). ${ }^{37,38}$ The two primary NRXN1 transcripts ( $\alpha 1$ and $\beta 1$ ) are shown in pink; other transcripts are not included. The long noncoding RNA (InRNA) AK127244 is shown in green. Each of the 22 exons is identified by a number according to the NM_004801.4 transcript. The five splice site (SS 1-5) locations are represented above the NRXN1- $\alpha$ transcript. The hollow pink box denoted by a P adjacent to each transcript represents the $\alpha$ promoter and $\beta$ promoter, respectively. All exonic and intronic deletions (chr2: 50,145,643-51,259,647; hg 19) are represented by solid red and yellow bars, respectively. Deletions with a black grid are subjects that were identified as having a second copy-number variation of potential clinical relevance. Inheritance status of the NRXN1 deletion is represented in brackets following the patient ID number (d.n., de novo; mat, maternal inheritance; pat, paternal inheritance; blank, unknown). P28 and P30 overlap both the $5^{\prime}$ and $3^{\prime}$ ends of NRXN1 and were not included in statistical analyses. P14 and P32 were identified as having maternal uniparental disomy of chromosome 14 and a RAF 1 mutation, respectively. The light blue box designates subjects with deletions overlapping exons numbered 5 and higher ( $3^{\prime}$ deletion). Case numbers were kept consistent throughout the article, tables, and supplemental documents. Cases P30, P34, and P35 were obtained from other laboratories and are represented in bold font. 
Rare exonic deletions overlapping NRXN1 on chromosome 2 p16 were first identified in individuals with autism spectrum disorder (ASD) $)^{11,12}$ and developmental delay/intellectual disability (DD/ID). ${ }^{13}$ Subsequently, such deletions have been identified in individuals with various neurodevelopmental and neuropsychiatric disorders. ${ }^{14-20} \mathrm{~A}$ few exonic NRXN1 deletions have been identified in controls, and the majority of transmitting parents are reported to be only mildly affected or clinically unaffected. ${ }^{10,14}$ Understanding the factors that contribute to this incomplete penetrance is a key goal for clinical genetics.

Exonic NRXN1 deletions are nonrecurrent and are found across the entire length of this large gene., ${ }^{2,10,21,22}$ There is conflicting evidence suggesting that the deletion extent may underlie the NRXN1 phenotypic heterogeneity. ${ }^{10,21}$ A recent study found that individuals with ASD are significantly more likely to harbor a rare de novo mutation in exons under purifying selection (called "critical exons") than their siblings, ${ }^{23}$ suggesting that certain sequences within NRXN1 may be more important for clinical expression than others. Also, accumulating evidence suggests that noncoding regions of the genome, including long noncoding RNAs (lncRNAs) and microRNAs (miRNAs), play an important role in the etiology of neurodevelopmental disorders. ${ }^{24,25}$ Deletions upstream and within introns of NRXN1 have been identified in disease cases; ${ }^{14,22,26}$ however, the pathogenicity of these deletions remains unclear.

As the use of genome-wide microarray technology in the prenatal setting increases, ${ }^{27}$ so does the need for an improved understanding of the genetic factors that impact the penetrance of NRXN1 deletions to inform genetic counseling and anticipatory care. We used a large $(n=19,263)$ clinically ascertained cohort from southern Ontario, Canada, and 15,264 populationbased controls to investigate the penetrance of NRXN1 deletions. Copy-number variation (CNV) data from high-resolution genome-wide microarrays allowed systematic evaluation of the burden of secondary CNVs that we used as a proxy to estimate the relative penetrance of exonic and intronic NRXN1 deletions.

\section{MATERIALS AND METHODS}

\section{Exonic NRXN1 deletions: clinical cohorts examined}

We searched three accredited Province of Ontario clinical cytogenetics laboratory databases for cases with exonic NRXN1 deletions. These included subjects submitted for clinical microarray testing before January 2015 at the Hospital for Sick Children $(n=11,727)$, Trillium Health Partners $(n=6,022)$, and Hamilton Health Sciences $(n=1,514)$, all located in southern Ontario, Canada. All three laboratories are provincially funded to provide clinical constitutional microarray testing for individuals with $\mathrm{DD} / \mathrm{ID}, \mathrm{ASD}$, and/or multiple congenital anomalies. The NRXN1 deletion cases identified in this study therefore have a strong ascertainment bias for these conditions. Participating physicians completed a detailed clinical checklist (Supplementary Table S1 online) for each exonic NRXN1 deletion case based on their own clinical assessment and lifetime chart reviews, when available. For cases without clinical checklist data, ascertainment and demographic data were collected from laboratory requisition forms. We also included three additional cases with an exonic NRXN1 deletion (P30, P34, and P35; Figure 1) detected by other clinical laboratories to increase the overall number of cases with genome-wide CNV data. These cases were not included in NRXN1 deletion prevalence calculations for the catchment area.

\section{Molecular methods and validation}

All exonic NRXN1 deletions (chr2:50,145,643-51,259,674; hg19) in cases were identified by one of three microarray platforms: the Affymetrix CytoScan HD Array, the Oxford Gene Technology (OGT) 4x180 CytoSure Oligonucleotide array, or the Illumina (formally BlueGnome) 4x180K CytoChip ISCA array. The OGT and Illumina arrays are similar in design with approximately 200-250 oligonucleotide probes (average probe spacing, 5.5-7.6 kb) distributed across the NRXN1 gene, allowing for a minimum deletion detection of $10 \mathrm{~kb}$. The CytoScan HD array has approximately 650 oligonucleotide probes distributed across NRXN1 with similar minimum deletion detection capabilities. All genomic coordinates are given using the Genome Reference Consortium February 2009 build of the human genome (GRCh37/hg 19). Genomic coordinates for the 22 NRXN1 exons ( $\alpha 1$ transcript; NM_004801.4) were obtained from the NCBI RefSeq database. Exonic NRXN1 deletions $\geq 100 \mathrm{~kb}$ were confirmed using fluorescence in situ hybridization (FISH). Smaller deletions were confirmed by quantitative polymerase chain reaction or by a second microarray.

\section{Additional clinically relevant CNVs as a proxy for calculating the relative penetrance of NRXN1 deletions}

All three clinical laboratories classified a deletion overlapping any one or more of the 22 NRXN1 exons (NM_004801.4) as pathogenic. Therefore, we used the presence of a second clinically relevant $\mathrm{CNV}$ as a proxy for estimating the relative penetrance of the NRXN1 deletions. As previously shown, ${ }^{28} \mathrm{CNV}$ s with high penetrance (i.e., not identified in control cohorts) are less likely to be accompanied by a second, large, rare $\mathrm{CNV}^{28}$ The high-resolution, genome-wide $\mathrm{CNV}$ data from each NRXN1 deletion case was investigated for the presence of a second CNV that may be clinically relevant. The clinical interpretation of these second CNVs as pathogenic, likely pathogenic, or as a variant of unknown significance (VUS) was provided by one of the three experienced clinical laboratory directors using the American College of Medical Genetics and Genomics guidelines for CNV interpretation. ${ }^{29}$ Other clinically relevant variants detected by different laboratory tests were not included in our statistical analyses.

\section{Phenotypic data}

The clinical checklist completed for each exonic NRXN1 deletion case is presented in Supplementary Table S1 online. Briefly, data were collected regarding ascertainment features, demographic variables (age, sex), growth parameters (height, weight, head circumference) and growth abnormalities, dysmorphic features, and lifetime developmental, psychiatric, medical, and family history based on clinical assessment and/or lifetime medical chart reviews. If completed, previous clinical genetic 
testing results were provided. When known, data regarding parental phenotypes were collected.

\section{Control cohorts}

To examine the prevalence of exonic and intronic NRXN1 deletions in the general population, we used 15,264 controls with $\mathrm{CNV}$ data available from high-resolution genome-wide arrays (Supplementary Table S3 online). These controls were analyzed on several different array platforms with variable probe spacing. Therefore, similar to previous studies, ${ }^{30}$ we only included exonic and intronic deletions identified by a minimum of two of three CNV calling algorithms (iPattern, PennCNV, ChAS) spanning five consecutive array probes and more than $10 \mathrm{~kb}$ in size. More than $90 \%$ of CNVs called using this method validate using other laboratory methods. ${ }^{30}$

\section{Intronic NRXN1 deletions}

Trillium Health Partners was the only clinical laboratory that systematically recorded intronic NRXN1 deletions. These clinical cases $(n=6,022)$ were analyzed using the Illumina $4 \mathrm{x} 180 \mathrm{~K}$ CytoChip ISCA array, which had even probe spacing across introns. We used two strategies to assess the pathogenicity of intronic NRXN1 deletions. First, we compared the prevalence of intronic NRXN1 deletions between clinically referred cases and the 15,254 population-based controls described. Second, we assessed the prevalence of secondary CNVs among the intronic NRXN1 deletion cases and compared this to the prevalence for exonic NRXN1 deletion cases.

\section{Statistical analyses}

Statistical analyses were performed using SAS software (version 9.2; SAS Institute, Cary, NC). For categorical data, we used $\chi^{2}$ or Fisher's exact test, as appropriate. For continuous data, we used the Welch $t$-test. All analyses were two-tailed, with statistical significance defined as $P<0.05$. Odds ratios (ORs) and $95 \%$ confidence intervals (CIs) were used to assess the prevalence of secondary CNVs between deletions overlapping one or more of exons 1-4 and those overlapping exons 5-22 and the association between the NRXN1 deletions (exonic and intronic) ascertained from the clinical diagnostic population compared with controls.

\section{RESULTS}

Prevalence of exonic NRXN1 deletions in cases and controls As of January 2015, a total of 19,263 individuals had been submitted for clinical microarray testing across the three participating cytogenetics laboratories. Forty-one $(0.21 \%)$ unrelated, previously unpublished, probands were identified as having deletions overlapping one or more NRXN1 exons (Figure 1). The prevalences of exonic NRXN1 deletions for the individual laboratories were $0.33,0.26$, and $0.14 \%$ (Supplementary Table S4 online). The prevalence of exonic NRXN1 deletions was significantly greater in cases compared with 15,264 controls (OR $=8.14$; 95\% CI: 2.91-22.72; $P<0.0001$ ) (Supplementary Table S3 online).

\section{Exonic NRXN1 deletions}

Genomic coordinates for all 44 exonic NRXN1 deletions, including three additional cases with exonic NRXN1 deletions from laboratories outside the catchment area, are presented in Figure 1. All were nonrecurrent, with sizes ranging from 29 to $806 \mathrm{~kb}$ (median $=244 \mathrm{~kb})$. The majority of the deletions $(n=32$; $72.7 \%$ ) overlapped at least one or more of exons $1-4$ (referred to as a $5^{\prime}$ NRXN1 deletion hereafter). Ten (22.7\%) deletions overlapped one or more of exons 5-22 (hereafter termed 3' NRXN1 deletions). Two deletions (P28 and P30; Figure 1) that overlapped both the $5^{\prime}$ and $3^{\prime}$ ends of NRXN1 were excluded from analyses comparing $5^{\prime}$ and $3^{\prime} N R X N 1$ deletions but not from the descriptive statistics. This categorization of exons has been used in previous publications. ${ }^{10,21}$ The $5^{\prime}$ NRXN1 deletions (median size $=242 \mathrm{~kb}$ ) were significantly larger than the $3^{\prime}$ NRXN1 deletions (median size $=83 \mathrm{~kb} ; \mathrm{t}(18.5)=2.55 ; P=0.019$ ).

The 44 exonic NRXN1 deletions were inherited (11 maternal, 5 paternal) in 16 (64.0\%), de novo in $9(36.0 \%)$, and unknown in 19 subjects. Of these, $5^{\prime}$ NRXN1 deletions were inherited in 13 (61.9\%) and de novo in $8(38.1 \%)$ subjects; $3^{\prime}$ NRXN1 deletions were inherited in $3(75.0 \%)$ and de novo in 1 (25.0\%) subject. Of the 11 transmitting mothers, 4 were identified as clinically affected: 3 with mild ID and 1 with anxiety and depression. Clinical outcomes for the remaining 7 transmitting mothers and 5 transmitting fathers were unknown.

\section{Genome-wide prevalence of additional clinically relevant CNVs}

Clinical microarray testing identified 10 (22.7\%) exonic NRXN1 deletion cases with one or more additional clinically relevant CNVs $(n=14)$ ranging in size from $38 \mathrm{~kb}$ to whole chromosomal anomalies (Table 1). The prevalence of males ( $n=7 / 28 ; 25.0 \%)$ with a secondary CNV was similar to that of females $(n=3 / 16 ; 18.8 \%)$. Twelve $(85.7 \%)$ of these CNVs were classified as VUS and two were classified as pathogenic (Table 1). The two pathogenic CNVs were de novo, two of the VUS were paternally inherited, and the remaining 10 additional VUS were of unknown inheritance. The prevalence of these secondary CNVs was significantly higher in subjects with a $3^{\prime}$ NRXN1 deletion $(n=7 / 10 ; 70.0 \%)$ compared with subjects with a $5^{\prime} N R X N 1$ deletion $(n=3 / 32 ; 9.4 \%$; OR $=7.47 ; 95 \%$ CI: 2.36-23.61; $P=0.0006$ ) (Figure 1). Eleven (78.6\%) of the 14 secondary CNVs overlapped one or more genes known to be involved in central nervous system function (Table 1).

\section{Clinical characteristics of exonic NRXN1 deletion cases}

Completed clinical checklists were returned for 21 (47.7\%) exonic NRXN1 deletion cases by referring physicians, with basic ascertainment and demographic data available for the other 23 cases (Supplementary Table S2 online). As expected, given the criteria for microarray testing, all of the 44 probands (16 female, 28 male) with exonic NRXN1 deletions were ascertained for DD/ID. Thirty-five (79.5\%) were children (median, 5.2; range, $1-6$ years) and 9 (20.5\%) were adults (median, 32.0; range, $21-59$ years). 


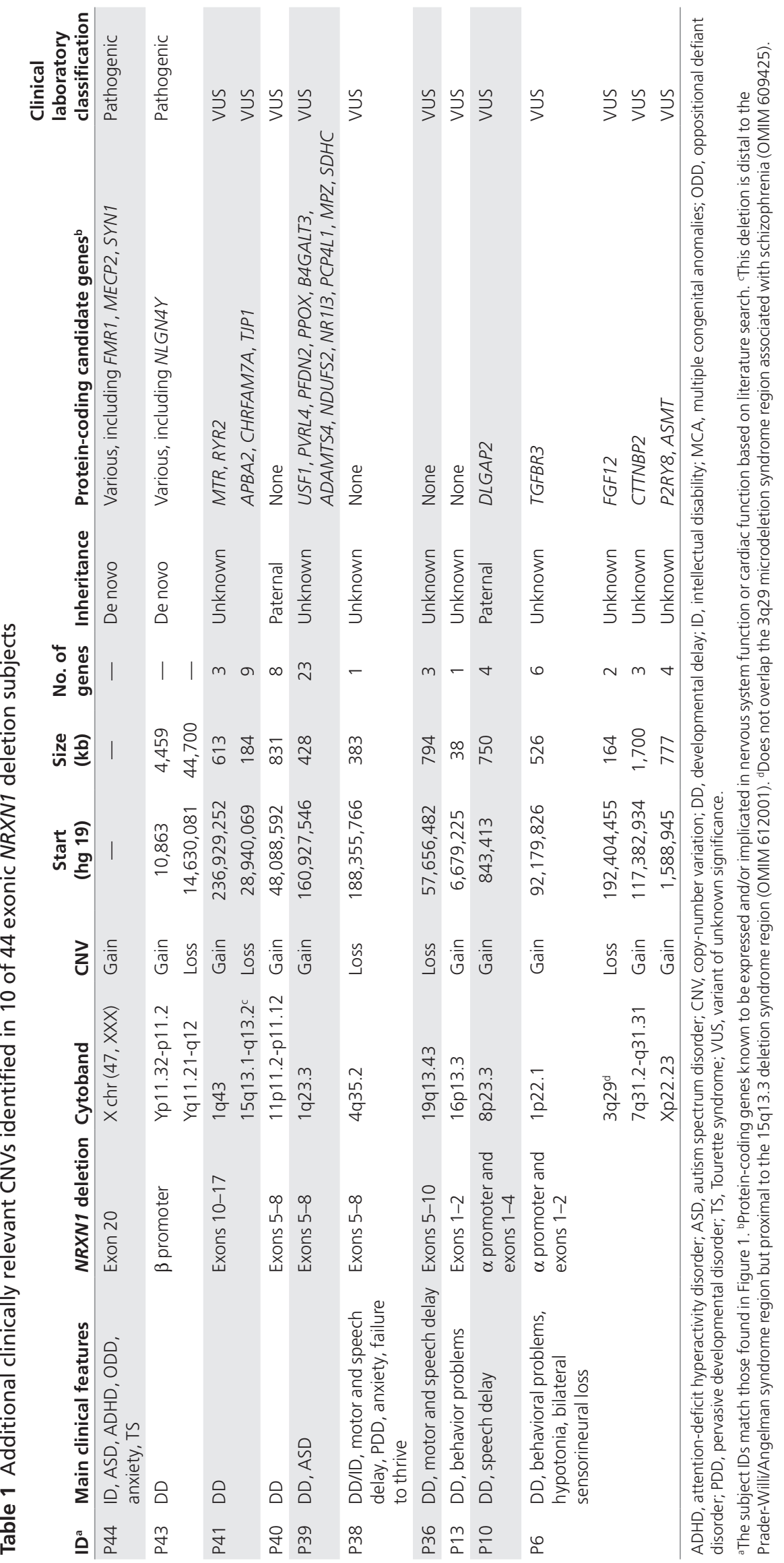




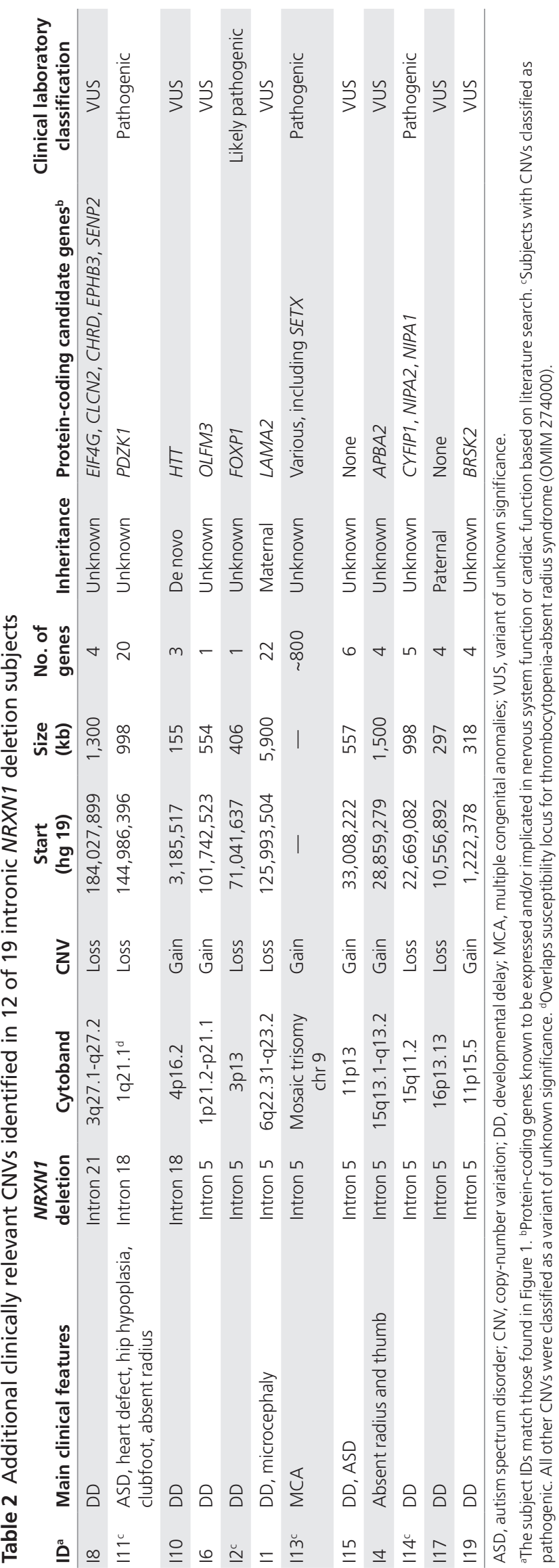

Twenty (45.5\%) subjects met diagnostic criteria for another (i.e., in addition to DD/ID) neurodevelopmental and/or neuropsychiatric condition, often referred to as "dual diagnosis." Including multiple features per subject, the prevalences of these conditions were as follows: ASD or pervasive developmental disorder $(n=14 ; 31.8 \%)$, epilepsy/seizures $(n=6 ; 13.6 \%)$, ADHD $(n=4$; $9.1 \%)$, anxiety ( $n=3 ; 6.8 \%)$, Tourette syndrome $(n=2 ; 4.5 \%)$, and tardive dyskinesia secondary to antipsychotic treatment in two adults with schizophrenia $(n=2 ; 4.5 \%)$. There was no significant difference in the prevalence of dual diagnosis, seizures/epilepsy, or macrocephaly between subjects with a $5^{\prime} N R X N 1$ deletion compared to subjects with a $3^{\prime} N R X N 1$ deletion (data not shown).

In contrast to the prominent neurodevelopmental phenotype, congenital anomalies were identified in only four (9.1\%) cases: two with tetralogy of Fallot (TOF), one of whom also had a tracheoesophageal fistula and imperforate anus; one with cryptorchidism; and one with a small atrial septal defect. All four of these subjects had a $5^{\prime}$ NRXN1 deletion and none were identified to have a rare second CNV. However, one TOF case was identified to have a $R A F 1$ sequence mutation causing Noonan syndrome (OMIM 611553).

Eleven $(25.0 \%)$ cases had additional clinical genetic testing with normal results with respect to karyotype, fragile X syndrome, 22q11.2 deletion syndrome, Prader-Willi syndrome, muscular dystrophy gene panel, and a gene panel for progressive myoclonic epilepsy. Two long contiguous stretches of homozygosity on chromosome 14 (identified on follow-up to be maternal uniparental disomy of chromosome 14) were identified in a single subject (P14; Figure 1).

\section{Intronic NRXN1 deletions}

We identified 19 of 6,022 (0.32\%) cases submitted for clinical microarray testing and 55 of 15,264 (0.36\%) controls with an intronic NRXN1 deletion (Figure 1). In contrast to the prevalence of exonic NRXN1 deletions identified in this laboratory (0.33\%; OR, 12.67; 95\% CI: 4.33-37.08; $P<0.0001)$, there was no significant difference in the prevalence of intronic NRXN1 deletions between cases and controls (OR 0.88; 95\% CI: 0.52$1.48 ; P=0.618)$. These intronic NRXN1 deletions ranged in size from 11 to $134 \mathrm{~kb}$ (median $=38 \mathrm{~kb}$ ), which is significantly smaller than the 44 exonic deletions $(P<0.0001)$. The majority ( $n=14 ; 73.7 \%)$ of the intronic deletions were located in the large intron 5 (Figure 1). Inheritance data are limited because most of the intronic deletions were not included in clinical reports; one case underwent follow-up and was found to be paternal in origin. Several of the intronic deletions were recurrent, including a 70.6-kb deletion in intron 5 and an 11.1-kb deletion in intron 18. Twelve (63.2\%) of the 19 intronic NRXN1 deletion cases had a second reportable CNV on clinical microarray (Table 2), which is a significantly greater prevalence than for the exonic NRXN1 deletion cases $(\mathrm{OR}=2.59 ; 95 \% \mathrm{CI}: 1.37-4.91 ; P=0.004)$.

Of these 19 (11 females, 8 males) unrelated cases with intronic NRXN1 deletions, 17 (89.5\%) were children (median $=5.0$; range $0.5-10$ years) and 2 were adults (Supplementary Table S2 online). Sixteen (84.2\%) were ascertained for DD/ID 
and/or ASD and $3(6.8 \%)$ were ascertained for multiple congenital anomalies $(n=1)$, CHD $(n=1)$, and absent radius/thumb. Of the 16 cases with DD and/or ASD, 10 (62.5\%) had a second rare $\mathrm{CNV}$ that overlapped one or more genes involved in central nervous system function (Table 2).

\section{DISCUSSION}

This is the largest study characterizing exonic and intronic NRXN1 deletions to date. Our aim was to use data from a large, clinical, population-based sample to systematically investigate factors affecting the penetrance of deletions overlapping this large gene. In addition to confirming the predominantly neuropsychiatric phenotypic expression of pathogenic CNVs overlapping $N R X N 1$, novel results support the importance of the genomic extent of these deletions, including the particular region involved and the exons overlapped.

\section{Penetrance of exonic NRXN1 deletions}

We report 44 novel exonic NRXN1 deletion cases ascertained for DD/ID (Supplementary Table S2 online). We assessed penetrance using multiple factors, both those used previously (prevalence in cases versus controls and the ratio of de novo versus inherited deletions) and those novel to this study (prevalence of secondary CNVs and their classification as pathogenic or VUS). We found that the $0.21 \%$ prevalence of exonic NRXN1 deletions in this clinical population was more than eightfold greater than in controls, and that $34 \%$ of these deletions were identified as being de novo, supporting the relatively high penetrance of these deletions for clinically important phenotypes. Approximately one in every four cases with an exonic NRXN1 deletion had one or more other rare CNVs reported on clinical microarray (Table 1 ). Their distribution among the cases indicated a relatively lower penetrance of 3' NRXN1 deletions.

\section{The effect of genomic position on penetrance of exonic NRXN1 deletions}

As in previous studies, ${ }^{10,21,22,31}$ the majority of the exonic $N R X N 1$ deletions identified in clinical cases overlapped the promoter and the first few exons of the NRXN1- $\alpha$ transcript. For subjects with deletions overlapping the $3^{\prime}$ end of NRXN1, there was a more than sevenfold higher likelihood of having a second clinically relevant CNV compared with subjects with a $5^{\prime}$ NRXN1 deletion (Figure 1). Furthermore, none of the additional rare CNVs identified in the $5^{\prime} N R X N 1$ deletion subjects was classified as pathogenic, demonstrating the relative importance of $5^{\prime} N R X N 1$ deletions over $3^{\prime} N R X N 1$ deletions for the expression of clinical phenotypes.

A potential explanation for the higher penetrance of $5^{\prime} N R X N 1$ deletions may be that these deletions directly overlap or indirectly influence the lncRNA AK127244 that is adjacent to the promoter of NRXN1- $\alpha$ (Figure 1). Accumulating evidence suggests that AK127244 plays a role in the etiology of neuropsychiatric disorders. ${ }^{26,32,33}$ This includes the identification of two deletions that overlap this lncRNA (and not
NRXN1) in a child with borderline IQ and early-onset schizophrenia. ${ }^{32}$ A recent report also described five ASD cases with deletions overlapping AK127244 (ref. 26). The biological function of AK127244 has yet to be elucidated. However, a significant proportion of lncRNAs are expressed in the brain and have important roles in neurodevelopmental processes. ${ }^{34}$

There was little evidence that additional phenotypic features were indicators of higher penetrance in individuals with exonic NRXN1 deletions in this study. This could be due in part to a high phenotypic floor effect present in clinically recruited subjects. It is interesting that the two adults with comorbid ID and schizophrenia in this study had deletions overlapping the $5^{\prime}$ end of NRXN1. Similarly, in the largest study of NRXN1 deletions in schizophrenia to date, 10 exonic NRXN1 deletions were identified, the majority of which overlapped the $5^{\prime}$ end of $N R X N 1 .{ }^{14}$ Also, two cases from that study had a deletion overlapping AK127244 but not NRXN1, ${ }^{14}$ providing support for the possible role of this lncRNA in the etiology of schizophrenia.

In contrast to our findings supporting the reduced penetrance of $3^{\prime}$ NRXN1 deletions, the limited number of these deletions reported in the literature had previously led to the hypothesis that they are associated with severe phenotypes, including prenatal lethality. ${ }^{31}$ To further investigate whether deletions overlapping the $3^{\prime}$ end of NRXN1 are associated with prenatal lethality, we examined 10 studies of miscarriages and/ or stillbirths (see refs. 6 and 17-26 in Rosenfeld et al. ${ }^{35}$ ) for rare CNVs overlapping NRXN1. Among more than 900 products of conception, only one paternally inherited $95-\mathrm{kb}$ deletion overlapping NRXN1- $\alpha$ (exons 2-4) was identified. ${ }^{35}$ We also identified one deletion overlapping the $N R X N 1-\beta$ promoter among 15,254 controls. These data provide further support for the increased penetrance of $5^{\prime}$ relative to $3^{\prime} N R X N 1$ deletions.

\section{Interpretation of intronic NRXN1 deletions}

We identified 19 novel cases with an intronic NRXN1 deletion: 16 with DD/ID and/or ASD and 3 with a congenital anomaly. Our group used two strategies to assess the penetrance of intronic NRXN1 deletions. First, we compared the prevalence of intronic NRXN1 deletions between cases and population-based controls and detected no significant difference. Indeed, 18 of the 19 intronic NRXN1 deletions identified in cases had breakpoints similar to those seen in controls (Figure 1). Second, we determined that intronic $N R X N 1$ deletion cases were twice as likely to harbor a second clinically relevant $\mathrm{CNV}$ compared with exonic NRXN1 deletion cases. Therefore, intronic NRXN1 deletions appear unlikely to substantially increase the risk for a neurodevelopmental disorder and/or multiple congenital anomalies.

However, seven (36.8\%) of the intronic NRXN1 deletion cases, each with a severe phenotype, had no additional rare $\mathrm{CNV}$ reported on clinical microarray. One of these seven NRXN1 deletions overlapped intron 9 (case I16; Figure 1), which had no corresponding deletion identified in controls. Using the VISTA enhancer browser (https://enhancer.lbl.gov), ${ }^{36}$ we identified a known enhancer element (hs1348) located $37 \mathrm{~kb}$ 
upstream of the intron 9 deletion that, if perturbed, could potentially alter the transcriptional levels of NRXN1 and thus increase the penetrance of this deletion.

\section{Advantages and limitations}

There are several advantages to this study. We used data from three clinical laboratories to compile the largest cohort of clinically referred individuals with exonic and intronic NRXN1 deletions assembled to date. Our systematic approach to the detection and interpretation of additional rare CNVs allowed us to use the burden of these secondary CNVs as a proxy for determining the relative penetrance of NRXN1 deletions. We used robust methods for CNV detection to evaluate the prevalence of intronic NRXN1 deletions in cases and controls that had previously been ignored due to the use of different $\mathrm{CNV}$ calling algorithms and reporting practices across clinical laboratories.

A limitation of this study is that the prevalence of individual clinical features would necessarily be influenced by the ascertainment bias inherent in clinically referred cases. This tends to overestimate $\mathrm{DD} / \mathrm{ID}$ and underestimate other features. The prevalence of NRXN1 deletions was approximately an order of magnitude different between each clinical laboratory, reaching statistical significance between the Hospital for Sick Children (0.14\%) and Trillium Health Partners (0.33\%) (OR 2.29; 95\% CI: 1.19-4.39; $P=0.012$ ) (Supplementary Table S3 online). This may be due to differences in indications for referral, with the Hospital for Sick Children and Hamilton Health Sciences servicing children with the most severe and intractable neurological disorders from this catchment area. Given that some NRXN1 deletions are identified in control subjects, ${ }^{14,31}$ it is possible that the prevalence of these deletions is highest at Trillium Health Partners because it is a community-based hospital that services individuals with comparatively milder clinical phenotypes. The resolution of the microarrays used to define the NRXN1 deletion breakpoints and detect genome-wide structural variants limited us to those $>10 \mathrm{~kb}$ that could influence phenotypic expression.

Only one control cohort (OPGP; Supplementary Table S3 online) was systematically screened for neurodevelopmental and/or neuropsychiatric conditions. Thus, control subjects with an exonic or intronic NRXN1 deletion may have had mild and/ or subclinical symptoms. This could have reduced the effect size of the case-control results. We did not have access to the individual SNP data for the NRXN1 deletion cases or the controls, we were therefore unable to genetically confirm that each individual was unrelated to any other. However, given that the exonic NRXN1 deletions identified in cases or controls had no similar breakpoints, it is unlikely that these individuals were related. Furthermore, only two of the eight control cohorts (Supplementary Table S3 online) were ascertained from the same catchment area as our cases, and they included only adults. This makes it unlikely that the intronic NRXN1 deletion cases ( $89 \%$ children) were the same individuals as those among the controls.

\section{Future directions}

The major challenge moving forward will be to determine how genetic and nongenetic factors converge to explain the variable expression and incomplete penetrance of exonic and intronic NRXN1 deletions. Examining the genes overlapped by additional rare CNVs as well as applying next-generation sequencing to detect variants within the coding and noncoding regions of the genome in subjects with a NRXN1 deletion may serve as key steps toward identifying novel pathways to disease expression. Interestingly, none of the second rare CNVs identified in this study has previously been reported in combination with an exonic NRXN1 deletion, ${ }^{10,21,22,31}$ suggesting that a large NRXN1 deletion consortium may be required to compile enough cases to identify novel biological patterns among the additional variants.

\section{Conclusions}

The expression of exonic NRXN1 deletions appears to be primarily neuropsychiatric, with DD/ID often comorbid with another neuropsychiatric condition. The results of this study suggest that deletions near the $5^{\prime}$ end of NRXN1 have higher penetrance, potentially related to perturbation of the lncRNA AK127244 located adjacent to the NRXN1- $\alpha$ promoter. Subjects with a $3^{\prime}$ NRXN1 deletion had a sevenfold increased likelihood of having a second rare CNV detected by clinical microarray, supporting reduced penetrance for these deletions. There is insufficient evidence to suggest that all intronic NRXN1 deletions are benign.

\section{SUPPLEMENTARY MATERIAL}

Supplementary material is linked to the online version of the paper at http://www.nature.com/gim

\section{ACKNOWLEDGMENTS}

The authors thank the patients and their families for their participation and Dr. Gregory Costain for his critical review of the manuscript. Study support was provided by the Canadian Institutes of Health Research (MOP 111238 and MOP 89066 to A.S.B.). A.S.B. holds the Canadian Research Chair in Schizophrenia Genetics and Genomic Disorders and the Dalglish Chair in 22q11.2 Deletion Syndrome. C.L. is supported by a Frederick Banting and Charles Best CIHR Doctoral Award. S.W.S. holds the GlaxoSmithKlineCIHR Endowed Chair in Genomic Sciences at the Hospital for Sick Children and University of Toronto. Acknowledgements for control data sets are in Supplementary Table S5 online.

\section{DISCLOSURE}

The authors declare no conflict of interest.

\section{REFERENCES}

1. Siddiqui TJ, Pancaroglu R, Kang Y, Rooyakkers A, Craig AM. LRRTMs and neuroligins bind neurexins with a differential code to cooperate in glutamate synapse development. J Neurosci 2010;30:7495-7506.

2. Tabuchi K, Südhof TC. Structure and evolution of neurexin genes: insight into the mechanism of alternative splicing. Genomics 2002;79:849-859.

3. Püschel AW, Betz $\mathrm{H}$. Neurexins are differentially expressed in the embryonic nervous system of mice. J Neurosci 1995;15:2849-2856. 
4. Zeng Z, Sharpe CR, Simons JP, Górecki DC. The expression and alternative splicing of alpha-neurexins during Xenopus development. Int J Dev Biol 2006;50:39-46.

5. Ushkaryov YA, Hata Y, Ichtchenko K, et al. Conserved domain structure of beta-neurexins. Unusual cleaved signal sequences in receptor-like neuronal cell-surface proteins. J Biol Chem 1994;269:11987-11992.

6. Koehnke J, Katsamba PS, Ahlsen G, et al. Splice form dependence of beta-neurexin/neuroligin binding interactions. Neuron 2010;67:61-74.

7. Boucard AA, Chubykin AA, Comoletti D, Taylor P, Südhof TC. A splice code for trans-synaptic cell adhesion mediated by binding of neuroligin 1 to alpha- and beta-neurexins. Neuron 2005;48:229-236.

8. Uemura T, Lee SJ, Yasumura M, et al. Trans-synaptic interaction of GluRdelta2 and Neurexin through Cbln1 mediates synapse formation in the cerebellum. Cell 2010;141:1068-1079.

9. Sugita S, Saito F, Tang J, Satz J, Campbell K, Südhof TC. A stoichiometric complex of neurexins and dystroglycan in brain. J Cell Biol 2001;154: 435-445.

10. Béna F, Bruno DL, Eriksson M, et al. Molecular and clinical characterization of 25 individuals with exonic deletions of NRXN1 and comprehensive review of the literature. Am J Med Genet B Neuropsychiatr Genet 2013;162B:388-403.

11. Szatmari $P$, Paterson $A D, Z$ waigenbaum $L$, et al. Mapping autism risk loci using genetic linkage and chromosomal rearrangements. Nat Genet 2007;39: 319-328.

12. Marshall CR, Noor A, Vincent JB, et al. Structural variation of chromosomes in autism spectrum disorder. Am J Hum Genet 2008;82:477-488.

13. Zahir FR, Baross A, Delaney AD, et al. A patient with vertebral, cognitive and behavioural abnormalities and a de novo deletion of NRXN1 alpha. J Med Genet 2008;45:239-243.

14. Rujescu D, Ingason A, Cichon S, et al.; GROUP Investigators. Disruption of the neurexin 1 gene is associated with schizophrenia. Hum Mol Genet 2009;18:988-996.

15. Nag A, Bochukova EG, Kremeyer B, et al.; Tourette Syndrome Association International Consortium for Genetics. CNV analysis in Tourette syndrome implicates large genomic rearrangements in COL8A1 and NRXN1. PLoS One 2013;8:e59061.

16. Møller RS, Weber YG, Klitten LL, et al.; EPICURE Consortium. Exon-disrupting deletions of NRXN1 in idiopathic generalized epilepsy. Epilepsia 2013;54: 256-264.

17. Noor A, Lionel AC, Cohen-Woods S, et al. Copy number variant study of bipolar disorder in Canadian and UK populations implicates synaptic genes. Am J Med Genet B Neuropsychiatr Genet 2014;165B:303-313.

18. McGrath LM, Yu D, Marshall C, et al. Copy number variation in obsessivecompulsive disorder and tourette syndrome: a cross-disorder study. J Am Acad Child Adolesc Psychiatry 2014;53:910-919.

19. Swaminathan S, Shen L, Kim S, et al.; Alzheimer's Disease Neuroimaging Initiative; NIA-LOAD/NCRAD Family Study Group. Analysis of copy number variation in Alzheimer's disease: the NIALOAD/ NCRAD Family Study. Curr Alzheimer Res 2012;9:801-814

20. Liu X, Cheng R, Ye X, et al. Increased rate of sporadic and recurrent rare genic copy number variants in Parkinson's disease among Ashkenazi Jews. Mol Genet Genomic Med 2013;1:142-154.
21. Schaaf CP, Boone PM, Sampath $S$, et al. Phenotypic spectrum and genotypephenotype correlations of NRXN1 exon deletions. Eur J Hum Genet 2012:20:1240-1247.

22. Curran S, Ahn JW, Grayton H, Collier DA, Ogilvie CM. NRXN1 deletions identified by array comparative genome hybridisation in a clinical case seriesfurther understanding of the relevance of NRXN1 to neurodevelopmental disorders. J Mol Psychiatry 2013;1:4.

23. Uddin M, Tammimies K, Pellecchia G, et al. Brain-expressed exons under purifying selection are enriched for de novo mutations in autism spectrum disorder. Nat Genet 2014:46:742-747.

24. Noor A, Whibley A, Marshall CR, et al.; Autism Genome Project Consortium. Disruption at the PTCHD1 locus on Xp22.11 in autism spectrum disorder and intellectual disability. Sci Trans/ Med 2010;2:49ra68.

25. Brzustowicz LM, Bassett AS. miRNA-mediated risk for schizophrenia in 22q11.2 deletion syndrome. Front Genet 2012;3:291

26. Walker S, Scherer SW. Identification of candidate intergenic risk loci in autism spectrum disorder. BMC Genomics 2013;14:499.

27. Hayden EC. Prenatal-screening companies expand scope of DNA tests. Nature 2014;507:19.

28. Girirajan S, Rosenfeld JA, Coe BP, et al. Phenotypic heterogeneity of genomic disorders and rare copy-number variants. N Engl J Med 2012;367:1321-1331.

29. Kearney HM, Thorland EC, Brown KK, Quintero-Rivera F, South ST; Working Group of the American College of Medical Genetics Laboratory Quality Assurance Committee. American College of Medical Genetics standards and guidelines for interpretation and reporting of postnatal constitutional copy number variants. Genet Med 2011;13:680-685.

30. Lionel AC, Crosbie J, Barbosa N, et al. Rare copy number variation discovery and cross-disorder comparisons identify risk genes for ADHD. Sci Trans/ Med 2011;3:95ra75

31. Dabell MP, Rosenfeld JA, Bader P, et al. Investigation of NRXN1 deletions: clinical and molecular characterization. Am J Med Genet A 2013;161A:717-731.

32. Duong LT, Hoeffding LK, Petersen KB, et al. Two rare deletions upstream of the NRXN1 gene (2p16.3) affecting the non-coding mRNA AK127244 segregate with diverse psychopathological phenotypes in a family. Eur J Med Genet 2015;58:650-653.

33. Pedrosa E, Kaushik S, Lachman HM. ChIP-chip analysis of neurexins and other candidate genes for addiction and neuropsychiatric disorders. J Neurogenet 2010;24:5-17

34. Briggs JA, Wolvetang EJ, Mattick JS, Rinn JL, Barry G. Mechanisms of long noncoding RNAs in mammalian nervous system development, plasticity, disease, and evolution. Neuron 2015;88:861-877.

35. Rosenfeld JA, Tucker ME, Escobar LF, et al. Diagnostic utility of microarray testing in pregnancy loss. Ultrasound Obstet Gynecol 2015;46:478-486.

36. Visel A, Minovitsky S, Dubchak I, Pennacchio LA. VISTA Enhancer Browser-a database of tissue-specific human enhancers. Nucleic Acids Res 2007;35(Database issue):D88-D92.

37. MacDonald JR, Ziman R, Yuen RK, Feuk L, Scherer SW. The Database of Genomic Variants: a curated collection of structural variation in the human genome. Nucleic Acids Res 2014;42(Database issue):D986-D992.

38. Zarrei M, MacDonald JR, Merico D, Scherer SW. A copy number variation map of the human genome. Nat Rev Genet 2015;16:172-183. 